\title{
Disappointing results of spring-assisted cranial vault expansion in patients with Crouzon syndrome presenting with sagittal synostosis
}

\author{
Alexander T. Wilson, BS, ${ }^{1}$ Linda Gaillard, MD, ${ }^{1}$ Sarah L. Versnel, MD, PhD, ${ }^{1}$ \\ Jochem K. H. Spoor, MD, ${ }^{2}$ Marie-Lise C. van Veelen, MD, PhD, ${ }^{2}$ and \\ Irene M. J. Mathijssen, MD, PhD'
}

Departments of ${ }^{1}$ Plastic and Reconstructive and Hand Surgery and ${ }^{2}$ Neurological Surgery, Erasmus Medical Center, Rotterdam, The Netherlands

\begin{abstract}
The aim of this study was to report on a single center's experience with spring-assisted cranial vault expansion (SAE) in patients with Crouzon syndrome and sagittal suture synostosis. Strip craniotomy with SAE has resulted in successful outcomes with low complication and revision rates in patients with isolated scaphocephaly. However, recent experience suggests that outcomes in patients with Crouzon syndrome and sagittal synostosis (SS) who undergo SAE are less favorable compared with the outcomes of those who undergo frontobiparietal (FBP) expansion. The authors reviewed both operations performed at a single center and noticed an upward expansion of the skull, which may be related to ventriculomegaly, with concurrent intracranial hypertension and poor aesthetic outcome. All patients diagnosed with Crouzon syndrome and SS who were treated with SAE required a revision FBP operation. Based on this outcome, the authors consider Crouzon syndrome a contraindication for correcting SS with springs.
\end{abstract}

https://thejns.org/doi/abs/10.3171/2021.1.FOCUS20739

KEYWORDS Crouzon syndrome; craniosynostosis; sagittal synostosis; ventriculomegaly

$\mathrm{S}$ PRING-ASSISTED cranial vault expansion (SAE) was first described by Lauritzen et al. in 1998 and has since been widely adopted by various craniofacial centers globally, including our own starting in 2010..$^{1-3}$ The advantages of this technique include early operative intervention and minimized blood loss and operative time, resulting in an improved morbidity profile compared with traditional whole-vault remodeling techniques. ${ }^{4} \mathrm{~A}$ drawback is the need for a second operation for device removal. Overall, SAE yields good functional and aesthetic results in the treatment of isolated sagittal craniosynostosis. ${ }^{5}$

At our center, the preferred management of sagittal synostosis (SS) involves scheduled SAE prior to 6 months of age. Prior to 2010, frontobiparietal (FBP) remodeling was performed at the age of 6 to 9 months. This switch in protocol was recently reviewed and showed SAE to be safe and effective, with reduced operative time, reduced blood loss, and a lower incidence of postoperative intracranial hypertension $(\mathrm{ICH}){ }^{5}$ However, recent observations suggest that SAE may be less effective in patients diagnosed with SS and Crouzon syndrome. We therefore evaluated surgical treatment in all patients with Crouzon syndrome and scaphocephaly due to SS.

\section{Methods}

The medical records of all patients with Crouzon syndrome treated at our center were reviewed. Only those with SS confirmed by CT were included in the analysis. The management of patients with Crouzon syndrome at our institution includes regular occipitofrontal head circumference (OFC) measurement, fundoscopy, polysomnography, and MRI of the brain. Data from all of these assessments were evaluated along with postoperative aesthetic outcomes (Table 1). Mild obstructive sleep apnea was diagnosed by an obstructive apnea-hypopnea index (oAHI) greater than 1 , and oAHI greater than 5 diagnosed moderate to severe disease. Skull growth restriction was indicated by a drop of 0.5 standard deviations in OFC measurements indexed to each patient's own progression curve over 2 years. The cranial index (CI) was also calculated

ABBREVIATIONS CI = cranial index; FBP = frontobiparietal; ICH = intracranial hypertension; ICP = intracranial pressure; oAHI = obstructive apnea-hypopnea index; OFC = occipitofrontal head circumference; SAE = spring-assisted cranial vault expansion; $\mathrm{SS}$ = sagittal synostosis.

SUBMITTED September 16, 2020. ACCEPTED January 12, 2021.

INCLUDE WHEN CITING DOI: 10.3171/2021.1.FOCUS20739. 


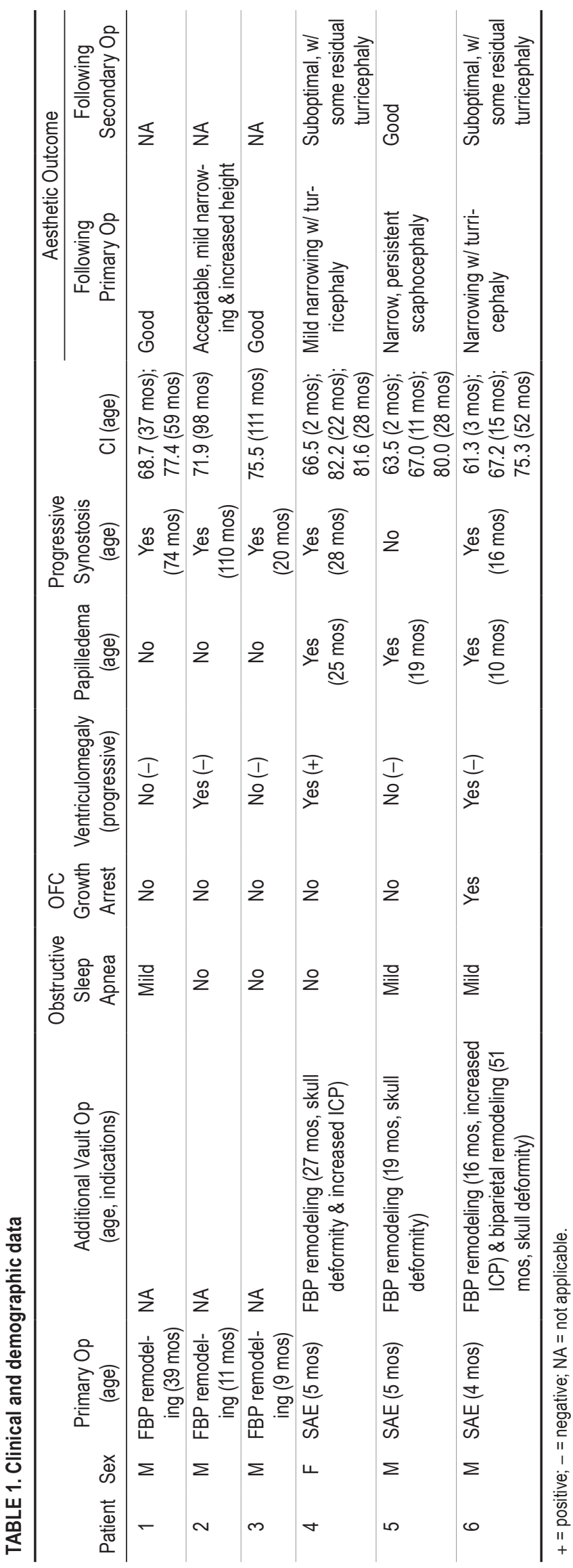

A
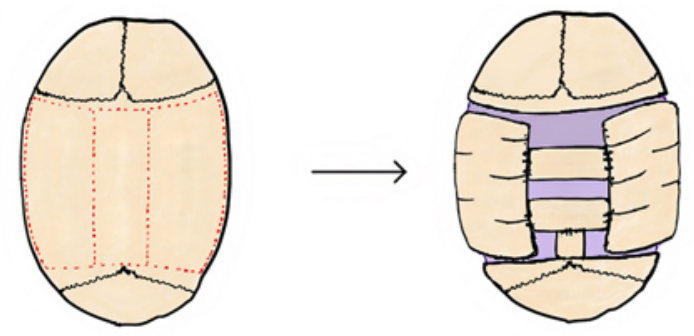

B
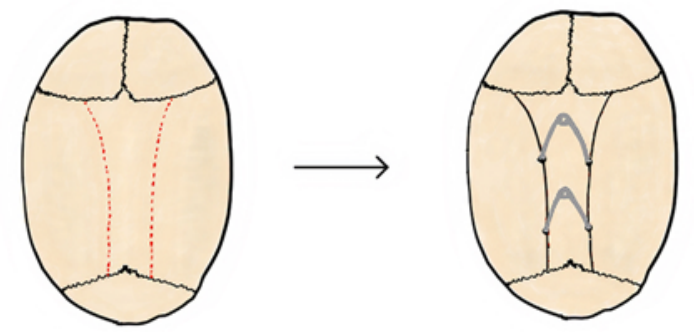

FIG. 1. A: FBP expansion. Osteotomies are shown as dashed red lines, and the bone flap design is shown to the right. B: Spring-assisted expansion. Osteotomies are shown as dashed red lines, and subsequent spring placement is shown to the right. Copyright Alexander T. Wilson. Published with permission.

pre- and postoperatively at the earliest date of available radiographic, CT, or MRI data. The CI was determined by dividing the maximum skull breadth by the maximum skull length and multiplying by 100 .

\section{Operative Technique}

Osteotomies varied significantly between techniques. Two parasagittal osteotomies were performed in SAE, with a bone strip left in place to avoid damage to the sagittal sinus. The widths of the osteotomies were tailored to the shape of each patient's skull but always within 3 to 4 $\mathrm{cm}$. In each patient, we used 9-N springs with a maximal width of $8.9 \mathrm{~cm}$ (BS 2056, grade 316S42, 1.22-mm thick; The Active Spring Co.), and spring removal was scheduled 12 weeks following placement. ${ }^{5}$ Osteotomies made during FBP operations also included parasagittal cuts, but the bone strip overlying the sagittal sinus was rotated $90^{\circ}$ to achieve biparietal widening (Fig. 1). The remaining bone graft was used to close the vault as much as possible. This technique has previously been described in detail. ${ }^{6}$

\section{Results}

In total, 6 patients with Crouzon syndrome were identified who underwent surgical treatment for SS (1999-2018). Three patients underwent a traditional FBP technique, and 3 were treated by SAE. None of the FBP-treated patients required additional vault expansion or revision (Table 1). All of the patients treated with SAE required a secondary FBP operation, and 1 (patient 6) required tertiary biparietal remodeling. In each patient, the indication for revision surgery was poor aesthetic outcome, with additional concerns for increased intracranial pressure (ICP) in some patients based on the results of fundoscopy and MRI. Ventriculomegaly occurred in 2 patients who underwent SAE and 1 who underwent FBP remodeling. Only 1 of these 

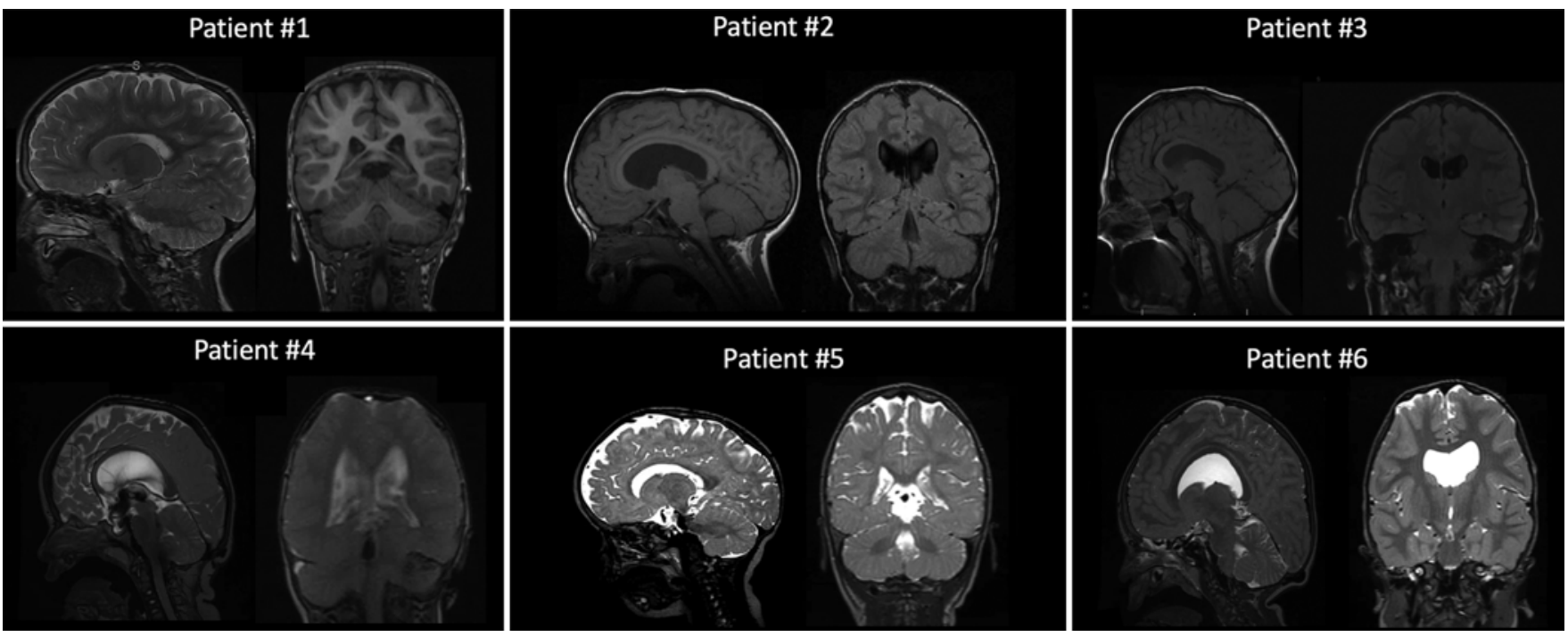

FIG. 2. Postoperative MR images obtained in each patient.

patients (patient 4) demonstrated progressive ventriculomegaly over 1 year following initial cranial vault expansion. CIs were similar in patients treated with FBP remodeling and SAE (Table 1). Progressive synostosis, which is common in patients with Crouzon syndrome, ${ }^{7}$ occurred in 4 patients at varying time intervals following vault expansion, but it did not appear to affect surgical outcome. The average time to progressive synostosis, when present, was 36 months, and the average time to surgical revision with SAE was 16 months.

Turricephalic growth and ventriculomegaly were observed in patients from both treatment groups (patients 2, 4 , and 6), but only those treated by SAE required revision surgery. In patient 4 , ventriculomegaly was noted prior to surgery and subsequent expansion occurred most dramatically in a cranial direction, with an upward lift of the sagittal bone strip. Good correction of the occiput was observed postoperatively, but biparietal narrowing and elevation of the vertex contributed to a suboptimal aesthetic outcome. Hydrocephalus was then diagnosed on subsequent imaging and confirmed by direct ICP monitoring 17 months following spring removal. FBP remodeling was performed, and biparietal widening was achieved with some residual turricephaly. No treatment (e.g., shunt placement) for ventriculomegaly was performed prior to the FBP operation.

In patient 5 , the features of Crouzon syndrome were not evident at the time of SAE. Intraoperatively, the parietal bones were observed to be thicker and less pliable than normal, perhaps limiting lateral expansion. A de novo unclassified variant in FGFR2 was then detected (c.931G>T, p.Val311Phe). Residual occipital bulleting was observed following SAE, and the parents mentioned that the patient had difficulty maintaining a supine position according to postoperative protocol. In the following months, nonspecific signs of increased ICP were noted (e.g., elongated optic nerve, large amount of CSF in the optic nerve sheath, globe flattening bilaterally), along with papilledema. Ultimately, FBP remodeling was performed for definitive correction of persistent skull narrowing and increased ICP.
The final aesthetic outcome was good, with notable biparietal widening and normalization of ICP.

Adequate correction of the occiput was achieved in patient 6 , with a fair amount of biparietal widening following initial SAE. Shortly thereafter, upward growth of the skull through the parasagittal osteotomies was noted, resulting in severe turricephaly along with signs of increased ICP, ventriculomegaly, and papilledema. Subsequently, FBP remodeling was performed, with biparietal narrowing still observed postoperatively. This was followed by additional biparietal remodeling 3 years later in an attempt to further correct the turricephalic shape. The final aesthetic result remains suboptimal due to residual turricephaly. Postoperative MR images obtained in each patient are shown in Fig. 2.

\section{Discussion}

Of the 6 patients with Crouzon syndrome and SS reviewed here, all 3 who underwent a primary SAE procedure before the age of 6 months also underwent a secondary cranial vault surgery. The indications for reoperation were inadequate skull deformity correction and development of ICH in the follow-up period. Although the benefits of SAE for patients with isolated SS include reduced $\mathrm{ICH}$, good aesthetic correction, and less blood loss, the revision rate in this initial cohort suggests that SAE is suboptimal in patients with Crouzon syndrome and SS. The underlying tendency toward ventricular expansion and progressive synostosis known to occur in patients with Crouzon syndrome, as well as the younger age at operation of the SAE-treated patients, may explain these findings.

Ventriculomegaly occurs in nearly half of all Crouzon patients and may be a benign dilation or progress to hydrocephalus requiring shunt placement. ${ }^{8-10}$ Progressive ventriculomegaly was seen in only 1 patient (patient 4 ), who was later confirmed to have hydrocephalus and increased ICP by direct measurement. Ventricular dilation following cranial vault expansion has been reported in patients with 
no preoperative evidence of hydrocephalus and attributed to a subclinical elevation in ICP, which is decompressed during surgery. ${ }^{8,11}$ Because postoperative ventricular dilation may occur, it seems plausible that the direction of its expansion, when present, may be influenced by the type of operation.

Following SAE, subsequent brain growth likely encounters differential resistance biparietally and vertically due to fewer osteotomies and reliance on progressive expansion. This dynamic approach retains physiological resistance at uninvolved sutures (e.g., squamosal), where the parietal bones are anchored but the difference between physiological resistance and that of the newly expanding osteotomies may direct brain growth upward toward the path of least resistance. In patients treated by FBP remodeling, cranial vault expansion is immediate and fixed, resulting in a relatively equal distribution of resistance because the cranial vault is reconstructed in a mosaic fashion following complete elevation of the parietal bone flaps. A greater degree of uniform resistance may be especially important in patients prone to postoperative ventricular dilation, such as those with Crouzon syndrome. In this way, if postoperative dilation occurs, it may result in uniform ventriculomegaly (as seen in patient 2 ) rather than ventriculomegaly marked by distortion of both ventricles and calvaria necessitating additional surgical correction (patients 4 and 6).

Additionally, dural attachments may play a role. The attachments to the central bone strip overlying the superior sagittal sinus are removed in only FBP remodeling because this segment is rotated $90^{\circ}$ to achieve biparietal widening. In SAE, these attachments are left in place with no change in the position of the strip. If abnormal flow through the superior sagittal sinus is present preoperatively due to cranial distortion, SAE would not address this and FBP remodeling would untether the bone strip, potentially resulting in a more physiological state.

Another factor that must be considered is the high rate of progressive synostosis in patients with Crouzon syndrome, which has been reported in up to $76.5 \%$ of cases. ${ }^{7}$ Postoperative lambdoid synostosis could result in occipital flattening and elevation of the vertex, thereby explaining the turricephaly observed in this study. In our cohort, lambdoid synostosis was noted in all patients treated with FBP remodeling and in 2 of 3 patients treated with SAE. Notably, the best aesthetic outcome in the SAE group was observed in patient 5, who experienced no progressive synostosis. However, if progressive lambdoid involvement was the only reason for the differences in outcomes between the SAE and FBP groups, we would expect higher rates of progressive synostosis in the SAE group than the FBP group, which is not the case. Therefore, it seems unlikely that progressive suture involvement fully explains the differences observed between the patients treated with FBP and SAE.

Last, age at surgical intervention may have influenced these outcomes, as SAE and FBP were performed at 4-5 months and 9-39 months of age, respectively. Skull growth occurs more rapidly in the first months of life, making it more difficult to achieve definitive correction early in development due to less predictable changes in postoperative growth. However, improved neuropsychological outcomes in patients treated before the age of 6 months have been reported. ${ }^{12}$ Therefore, we now prefer FBP remodeling at the age of 6 months for all patients with SS and Crouzon syndrome to provide timely relief of skull growth restriction and to attempt definitive correction of scaphocephaly.

\section{Conclusions}

We have briefly described our experience with SAE and FBP operations in patients with SS and Crouzon syndrome. We observed poor outcomes following SAE related to both the development of ICH during follow-up and head shape. Potential explanations for the worse outcomes observed in patients treated with SAE include ventriculomegaly, progressive synostosis, and age at the time of operation. Isolated SS rarely occurs in patients with Crouzon syndrome; therefore, the size of our study cohort is small. Further investigation of patients treated by both SAE and whole-vault remodeling techniques should continue in larger cohorts to elucidate the optimal treatment protocol for these patients. Based on our preliminary results, we have changed our protocol to perform FBP remodeling at 6 months of age for patients with Crouzon syndrome and $\mathrm{SS}$, and we recommend against the use of springs in these select patients.

\section{References}

1. Lauritzen C, Sugawara Y, Kocabalkan O, Olsson R. Spring mediated dynamic craniofacial reshaping. Case report. Scand J Plast Reconstr Surg Hand Surg. 1998;32(3):331-338.

2. David LR, Plikaitis CM, Couture D, et al. Outcome analysis of our first 75 spring-assisted surgeries for scaphocephaly. $J$ Craniofac Surg. 2010;21(1):3-9.

3. Taylor JA, Maugans TA. Comparison of spring-mediated cranioplasty to minimally invasive strip craniectomy and barrel staving for early treatment of sagittal craniosynostosis. J Craniofac Surg. 2011;22(4):1225-1229.

4. Arko L IV, Swanson JW, Fierst TM, et al. Spring-mediated sagittal craniosynostosis treatment at the Children's Hospital of Philadelphia: technical notes and literature review. Neurosurg Focus. 2015;38(5):E7.

5. van Veelen MC, Kamst N, Touw C, et al. Minimally invasive, spring-assisted correction of sagittal suture synostosis: technique, outcome, and complications in 83 cases. Plast Reconstr Surg. 2018;141(2):423-433.

6. van Veelen MLC, Mihajlović D, Dammers R, et al. Frontobiparietal remodeling with or without a widening bridge for sagittal synostosis: comparison of 2 cohorts for aesthetic and functional outcome. J Neurosurg Pediatr. 2015; 16(1):86-93.

7. Connolly JP, Gruss J, Seto ML, et al. Progressive postnatal craniosynostosis and increased intracranial pressure. Plast Reconstr Surg. 2004;113(5):1313-1323.

8. Cinalli G, Sainte-Rose C, Kollar EM, et al. Hydrocephalus and craniosynostosis. J Neurosurg. 1998;88(2):209-214.

9. Noetzel MJ, Marsh JL, Palkes H, Gado M. Hydrocephalus and mental retardation in craniosynostosis. J Pediatr. 1985; 107(6):885-892.

10. Proudman TW, Clark BE, Moore MH, et al. Central nervous system imaging in Crouzon's syndrome. J Craniofac Surg. 1995;6(5):401-405.

11. Collmann H, Sörensen N, Krauss J, Mühling J. Hydrocephalus in craniosynostosis. Childs Nerv Syst. 1988;4(5):279-285. 
12. Patel A, Yang JF, Hashim PW, et al. The impact of age at surgery on long-term neuropsychological outcomes in sagittal craniosynostosis. Plast Reconstr Surg. 2014;134(4):608e$617 \mathrm{e}$.

\section{Disclosures}

The authors report no conflict of interest concerning the materials or methods used in this study or the findings specified in this paper.

\section{Author Contributions}

Conception and design: all authors. Acquisition of data: all authors. Analysis and interpretation of data: all authors. Drafting the article: Mathijssen, Wilson, Gaillard, van Veelen. Critically revising the article: Mathijssen, Wilson, Gaillard, Spoor, van Veelen. Reviewed submitted version of manuscript: all authors. Statistical analysis: Wilson. Study supervision: Mathijssen, van Veelen.

\section{Correspondence}

Irene M. J. Mathijssen: Erasmus Medical Center, Rotterdam, The Netherlands.i.mathijssen@erasmusmc.nl. 